\title{
IMPLEMENTAÇÃO DE ESTRATÉGIA DE CONTROLE PID FUZZY EM REATOR CONTÍNUO DE CRAQUEAMENTO TÉRMICO
}

\author{
M. LAZZARI ${ }^{1}$, V. R. WIGGERS ${ }^{1}$, R. GOZDZIEJEWSKI Jr ${ }^{1}$, H. F. MEIER ${ }^{1}$ e L. ENDER ${ }^{1}$ \\ ${ }^{1}$ Universidade Regional de Blumenau, Departamento de Engenharia Química e Engenharia de \\ Alimentos \\ E-mail para contato: ender@furb.br
}

\begin{abstract}
RESUMO - Os controladores PID clássicos são bastante usados para controlar os processos industriais e assim permitir um melhor desempenho do mesmo e de forma segura e confiável. Sugere-se um estratégia de controle PID com ajuste on-line de seus parâmetros, baseados em lógica fuzzy, denominado PID fuzzy. A estratégia abordada tem como objetivo um melhor ajuste dos parâmetros do controlador, resultando uma qualidade operacional melhor e qualidade dos produtos obtidos superior. Apresenta-se neste trabalho a metodologia empregada, bem como um estudo comparativo do PID fuzzy e o PID clássico, aplicados em um reator contínuo de craqueamento térmico. Os experimentos foram conduzidos na temperatura de $550^{\circ} \mathrm{C}$, sendo provocadas perturbações na temperatura e na vazão de alimentação do reator, caracterizando problemas servo e regulador, respectivamente. Os resultados experimentais foram analisados através dos critérios de erro ISE, IAE e ITAE e do consumo de energia do reator. Com base nos experimentos realizados, verificou-se a eficiência da estratégia implementada, PID fuzzy, resultando em um controlador mais robusto.
\end{abstract}

\section{INTRODUÇÃO}

O conceito de conjunto fuzzy foi introduzido, em 1965, por Lotfi A. Zadeh (Universidade da Califórnia, Berkeley). A lógica fuzzy está relacionada com os princípios formais de raciocínio aproximado. A importância desta da lógica fuzzy vem do fato que a maioria dos modos de raciocínio humano está relacionado à inferência de respostas aproximadas para questões baseadas em conhecimentos inexatos, incompletas ou não totalmente confiáveis.

O desenvolvimento de um sistema de controle fuzzy é baseado na idéia de incluir o "conhecimento especialista" ou "experiência" de um operador para se obter a melhor estratégia de controle. A lógica fuzzy utiliza variáveis lingüisticas no lugar de variáveis numéricas. Variáveis lingüisticas admitem como valores apenas expressões lingüisticas (ou termos primários), que são representadas por conjunto fuzzy (Almeida et al., 2001).

Atualmente observam-se inúmeras indústrias necessitando de estratégias de controle em seus processos produtivos e, com isso, vem crescendo o incentivo pela implementação de estratégias de controle de muitos processos industriais complexos. Essas estratégias de controle avançadas requerem implementação dos algoritmos em microprocessadores e computadores para que seja possível ter 
ações mais rápidas e precisas quanto ao desempenho e segurança do processo. A utilização dos conceitos estabelecidos na lógica fuzzy no ajuste on-line dos parâmetros do controlador PID são passíveis de implementação em microprocessadores, permitindo assim embarcar a estratégia de controle proposta em controladores comerciais.

Um processo de craqueamento térmico é um processo termoquímico que decompõem a matéria orgânica, desmembrando as cadeias dos compostos, formando novos produtos (Wiggers, et al., 2013). $\mathrm{O}$ reator de craqueamento térmico opera em regime contínuo e apresenta comportamento altamente não linear, pois as reações de decomposição térmica são bastante complexas. $\mathrm{O}$ processo de craqueamento térmico sofre influência de diversas variáveis operacionais sendo uma unidade altamente interessante para avaliação de estratégias de controle (Lazzari, 2012).

\section{FUNDAMENTAÇÃO TEÓRICA}

Mesmo com o grande avanço de técnicas de controle avançado e sua tendência crescente de implementação na indústria de processos, é ainda muito comum a aplicação de técnicas de controle clássicas, especificamente o PID (Proporcional, Integral e Derivativo). Uma das possíveis razões para isso é a sua larga gama de aplicações e seu relativo baixo custo e facilidade de implantação quando comparado com técnicas de controle avançado. Levando-se isso em consideração neste trabalho, o controle clássico através dos controladores PID é também implementado e será comparado com o controle PID fuzzy. A estratégia de controle proposta está baseada no PID clássico, diferenciando-se por usar da lógica fuzzy no ajuste on-line dos parâmetros do controlador.

\subsection{Estratégia Clássica de Controle PID}

O algoritmo de controle PID é uma modalidade de controle clássico, onde a ação aplicada sobre uma variável manipulada é proporcional ao erro verificado entre o valor desejado e o medido. Três parcelas são consideradas: uma de proporcionalidade direta a cada novo erro, outra de proporcionalidade à soma acumulada do erro e uma terceira de proporcionalidade à taxa de variação do erro. O PID traduz a idéia de que a variável manipulada será alterada proporcionalmente ao erro que aparece a cada instante, ao acúmulo (integral) do erro ao longo do tempo e à taxa de variação do erro da variável controlada. Segundo Iserman (1989):

$$
u(k)=u(k-1)+q_{0} e(k)+q_{1} e(k-1)+q_{2} e(k-2)
$$

Onde:

$$
\begin{aligned}
& q_{0}=k_{c}\left(1+\frac{T_{0}}{2 \tau_{i}}+\frac{\tau_{d}}{T_{0}}\right) \\
& q_{1}=-k_{c}\left(1+2 \frac{\tau_{d}}{T_{0}}-\frac{T_{0}}{2 \tau_{i}}\right)
\end{aligned}
$$




$$
q_{2}=k_{c} \frac{\tau_{d}}{T_{0}}
$$

Desta forma o algoritmo de controle é conhecido como algoritmo de velocidade (Iserman, 1989).

\section{Ajustes do Controle PID Clássico}

Um dos procedimentos mais utilizados para o ajuste dos parâmetros PID baseia-se em procedimentos empíricos, os quais analisam o comportamento do sistema em malha fechada como aberta, variando-se as condições operacionais de entrada através de perturbações do tipo degrau, oscilatórias e outras.

Dentre os procedimentos de ajuste, destacam-se o método de Ziegler-Nichols, Cohen-Coon (Curva de Reação) e ITAE, dos quais o primeiro foi usado no neste trabalho. Estes procedimentos são descritos em detalhes em Seborg (1989), Sthephanopoulos (1984) e Ogata (2003).

0 valor de $K u$ é o valor do ganho proporcional que torna a saída do processo oscilante mantendo uma amplitude constante, e o $P u$ é o período desta oscilação. A determinação dos parâmetros $\mathrm{Ku}$ e $\mathrm{Pu}$ foram realizadas pela técnica proposta por Åström e Hägglund (1984), os quais desenvolveram uma técnica atrativa e alternativa para automatizar o método da curva de oscilação de Ziegler e Nichols. No teste auto-tuning via relê, um simples ensaio experimental é utilizado para determinar Ku e Tu.

\subsection{Controlador PID Fuzzy}

A estrutura PID fuzzy apresentada é uma alternativa interessante para tratar estes processos por realizar ajuste on-line dos parâmetros do PID. O sistema de inferência fuzzy é composto por um conjunto de regras que descrevem o comportamento desejado do controlador. O controlador PID fuzzy é expresso de forma similar ao PID clássico, diferenciando-se no ajuste on-line dos parâmetros do controlador $(K c, K i, K d)$, da seguinte forma:

$$
\begin{array}{ll}
K_{c}-\text { ganho Proporcional } \\
K_{i}=\frac{k_{c}}{\tau_{i}} & \text { (ganho Integral) } \\
K_{d}=k_{c} \cdot \tau_{d} & \text { (ganho Derivativo) }
\end{array}
$$

A Figura 1 apresenta o diagrama de blocos da estratégia de controle PID fuzzy, onde pode-se verificar que a estrutura da estratégia de controle está baseada na estratégia $P I D$ clássica, com ajustes on-line de seus parâmetros de acordo com um sistema de inferência fuzzy. 


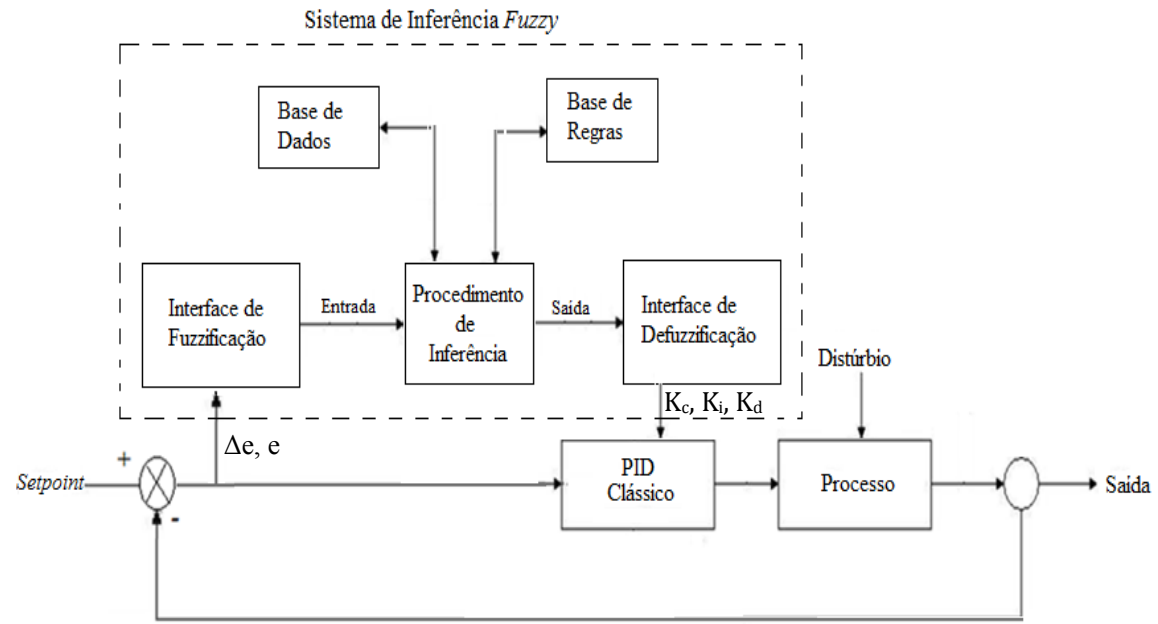

Figura 1: Estrutura de um controlador fuzzy (Fonte: SANDRI and CORREA, 1999).

\section{Sistema Fuzzy Implementado} de:

O sistema fuzzy implementado está fundamentada na proposta de Freitas et al. (2008), composto

a) 2 entradas: erro e taxa de variação do erro;

b) 3 saídas: $K_{c}, K_{i}$ e $K_{d}$;

c) Sistema 25 regras para cada saída;

d) Defuzzicação através do método do centro da área.

\section{Funções de Pertinência}

Na Figura 2 tem-se as funções de pertinência das variáveis de entrada, erro e taxa de erro. Nesta figura observam-se as seguintes nomenclaturas abaixo arroladas, as quais associam as respectivas variáveis linguísticas em parâmetros mensuráveis nas etapas de fuzzificação e defuzicação.

$\mathrm{BN}=$ grande negativo;

$\mathrm{SN}=$ pequeno negativo;

$\mathrm{EZ}=$ zero;

$\mathrm{SP}=$ pequeno positivo;

$\mathrm{BP}=$ grande positivo;

\section{$\underline{\text { Regras do Sistema Fuzzy }}$}

O conjunto de regras para o ajuste dos parâmetros $K_{c}, K_{i}$ e $K_{d}$ estão resumidas nas Tabelas 1 a 3 , respectivamente. Os pontos $\mathbf{P}$ e $\mathbf{G}$, representam os limites inferior e superior das variáveis de saída da lógica fuzzy $\left(\mathrm{K}_{\mathrm{c}}, \mathrm{K}_{\mathrm{i}}, \mathrm{K}_{\mathrm{d}}\right)$. O controlador PID que representa o ponto $\mathbf{G}$, apresenta ajuste de ZieglerNichols, aqui nominado de PID fast, e o controlador PID que representa o ponto $\mathbf{P}$ é um controlador mais conservativo (PID slow). 
Tabela 1:Regras deAjuste para o Ganho Proporcional $\left(K_{c}\right)$.

\begin{tabular}{|c|l|l|l|l|l|}
\hline $\boldsymbol{K}_{\boldsymbol{c}}$ & \multicolumn{5}{|c|}{ Erro } \\
\hline Taxa erro & BN & SN & EZ & SP & BP \\
\hline BN & G & G & G & G & G \\
\hline SN & G & G & P & G & G \\
\hline EZ & G & P & P & P & G \\
\hline SP & G & G & P & G & G \\
\hline BP & G & G & G & G & G \\
\hline \hline
\end{tabular}

Tabela 2:Regras de Ajuste para o Ganho Integral $\left(K_{i}\right)$.

\begin{tabular}{|c|l|l|l|l|l|}
\hline \hline $\boldsymbol{K}_{\boldsymbol{i}}$ & \multicolumn{5}{|c|}{ Erro } \\
\hline \hline Taxa erro & BN & SN & EZ & SP & BP \\
\hline BN & P & P & G & P & P \\
\hline SN & P & G & G & G & P \\
\hline EZ & P & G & G & P & P \\
\hline SP & P & G & G & G & P \\
\hline BP & P & P & G & P & P \\
\hline \hline
\end{tabular}

Tabela.3:Regras de Ajuste para o Ganho Derivativo $\left(K_{d}\right)$.

\begin{tabular}{|c|l|l|l|l|l|}
\hline \hline $\boldsymbol{K}_{\boldsymbol{d}}$ & \multicolumn{5}{|c|}{ Erro } \\
\hline \hline Taxa erro & BN & SN & EZ & SP & BP \\
\hline BN & P & P & P & P & P \\
\hline SN & G & P & P & P & G \\
\hline EZ & G & P & P & P & G \\
\hline SP & G & P & P & P & G \\
\hline BP & P & P & P & P & P \\
\hline \hline
\end{tabular}

Diante do apresentado nas tabelas anteriores, no sistema de inferência fuzzy implementado, o conjunto de regras e a etapa de defuzzicação é distinto para cada parâmetro de ajuste do controlador

\subsection{O Reator de Craqueamento Térmico}

O reator de craqueamento térmico utilizado nesse se encontra localizado no Laboratório de Desenvolvimento de Processos do Departamento de Engenharia Química e Engenharia de Alimentos da Universidade Regional de Blumenau.

O equipamento possui uma seção onde ocorre a reação de craqueamento e uma seção onde promove-se a condensação e recuperação dos produtos craqueados termicamente. Essa Figura é dividida em seções para melhor entendimento, portanto:

O reator possui seis termopares do tipo $K(T 1, T 2, T 3, T 4, T 5$ e $T 6)$, para medição das temperaturas na entrada, na saída e ao longo do reator. Possui um PT 100 (T7) para medir a 


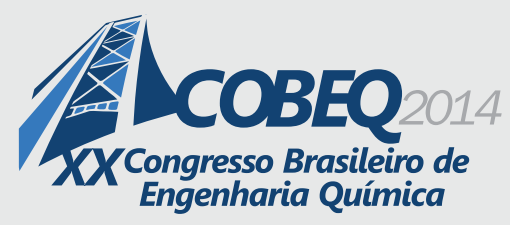

\section{9 a 22 de outubro de 2014 \\ Florianópolis/SC}

temperatura do condensado e também um sensor GTP 1000 para monitorar a pressão do sistema, este localizado na saída da bomba.

A seção onde ocorre a reação de craqueamento no reator é composta de resistências elétricas que aquecem a unidade e foi dividida em quatro malhas de controle distintas que tem o objetivo de favorecer a isotermicidade do sistema.

A primeira malha $(M 1$ ou $S S R P 1)$ é composta por três resistências elétricas de $350 \mathrm{~W}$ cada. A segunda malha (M2 ou SSRP2) possui duas resistências de $350 \mathrm{~W}$ cada. A terceira malha (M3 ou $S S R P 3)$ possui três resistências de $350 \mathrm{~W}$ cada e a quarta malha (M4 ou SSRP4) tem duas resistências de $350 \mathrm{~W}$ cada.

A unidade experimental possui um sistema SCADA ("Software Control and Data Aquisition") desenvolvido pela equipe do Laboratório LDP que possibilita a manutenção das condições operacionais através do controle das variáveis manipuladas e aquisição de todas as variáveis do processo aferidas durante o experimento.

\section{RESULTADOS E DISCUSSÕES}

O procedimento experimental adotado na execução dos ensaios é descrito a seguir: Após atingir e estabilizar a temperatura de setpoint do reator $\left(550^{\circ} \mathrm{C}\right)$, gera-se uma perturbação servo e uma perturbação regulador no processo. Para a perturbação servo incrementa-se em $20^{\circ} \mathrm{C}$ a temperatura de setpoint (perturbação I) e acompanha-se por aproximadamente 01 hora a evolução dos perfis de temperatura, em seguida, diminui-se a temperatura em $20^{\circ} \mathrm{C}$ no setpoint (perturbação II) e novamente aguarda-se 01 hora. Após isso, parte-se para a perturbação regulador, onde diminui-se a vazão de alimentação do reator de 283,4 g/h para 197,9 g/h (perturbação III); aguarda-se 01 hora para então retornar a vazão de alimentação inicial (perturbação IV) e observa-se novamente a evolução dos perfis por 01 hora. Após a estabilização desta última etapa finaliza-se o experimento.

A título de comparação foram gerados experimentos considerando a sequência de perturbações descritas, para os controladores PID slow, PID fast e PID fuzzy. Os dois primeiros representam os limites dos parâmetros $\left(K_{c}, K_{i}, K_{d}\right)$ usados na lógica fuzzy. Seguem nas Figuras 2 a 4 os resultados das respostas das malhas de controle e ações de controle, para os três controladores avaliados.

Diante dos resultados dos experimentos apresentados e com os dados plotados na Tabela 4, observa-se em relação ao PID slow um ganho na eliminação de erro pequenos (IAE menor) e erros que persistem no tempo (ITAE menor). Porém este desempenho não é verificado na eliminação de grandes erros. De uma forma geral, o desempenho do PID slow e PID fuzzy foram semelhantes nestas condições de operação. Observa-se as ações de controle mais enérgicas nas regiões de maior erro, o que está previsto nas regras fuzzy implementadas. Os resultados dos experimentos para o PID fast, mostram um comportamento oscilatório das variáveis manipuladas e ações enérgicas na carga térmica do reator. Os parâmetros do PID fast são os ajustados pela metodologia proposta por Ziegler-Nichols (Ogata, 1994), num procedimento de auto-tuning, com a determinação de $K u$ e $P u$ pela técnica proposta por Åström e Hägglund (1984). Independente da discussão se o auto-tuning mostrou-se 
eficiente, pode-se afirmar que o PID fuzzy aproximou-se bastante da resposta proporcionada pelo PID slow, buscando um comportamento mais robusto.

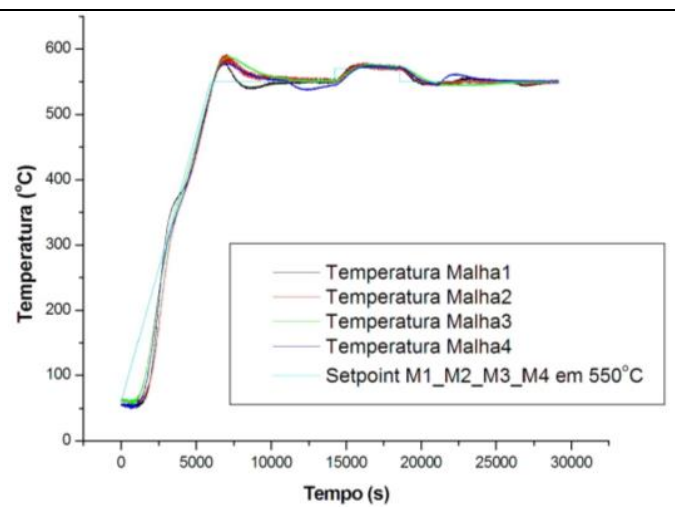

(a)

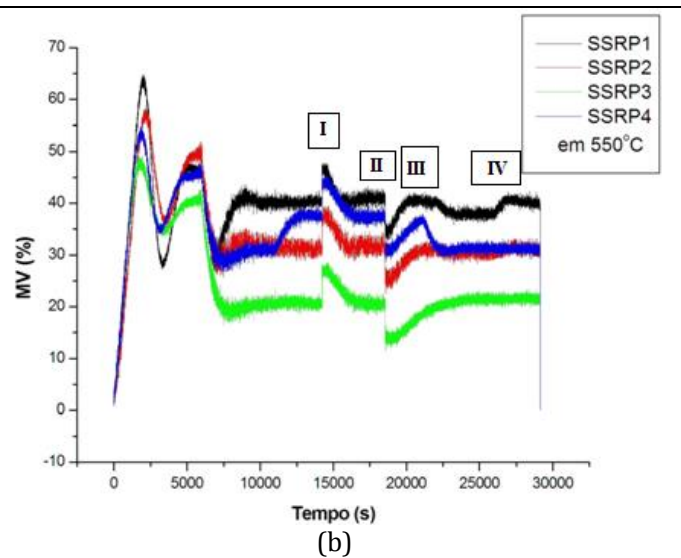

(b)

Figura 2: Temperaturas para as malhas de controle e ações de controle para PID slow.
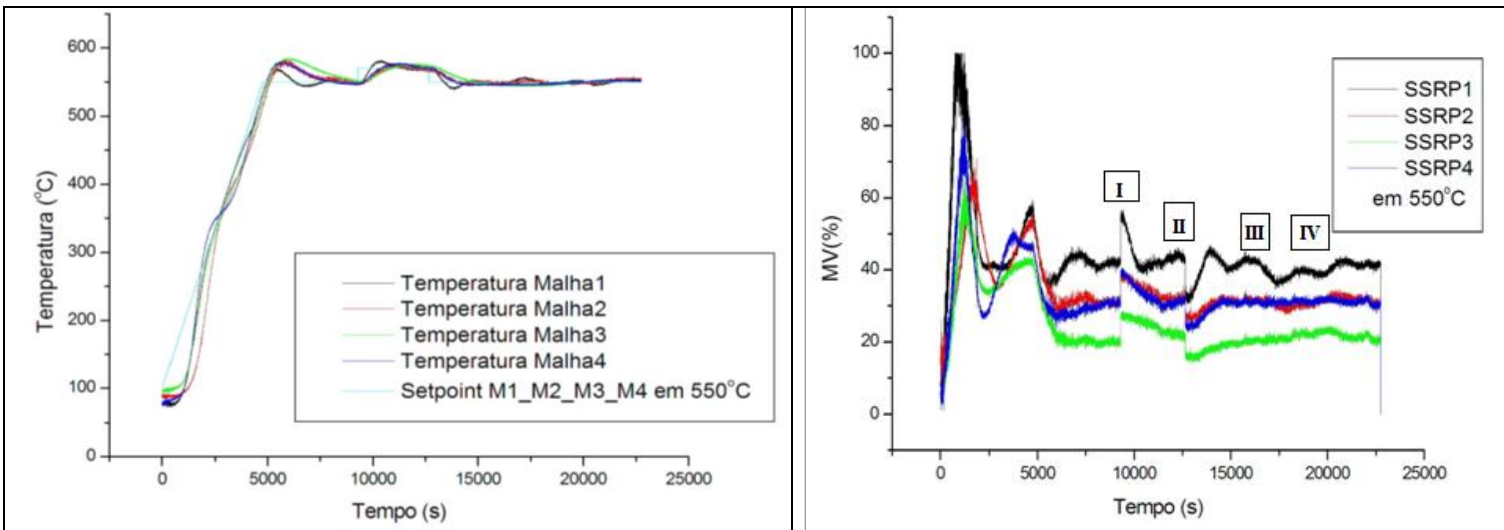

Figura 3: Temperaturas para as malhas de controle e ações de controle para PID fuzzy.

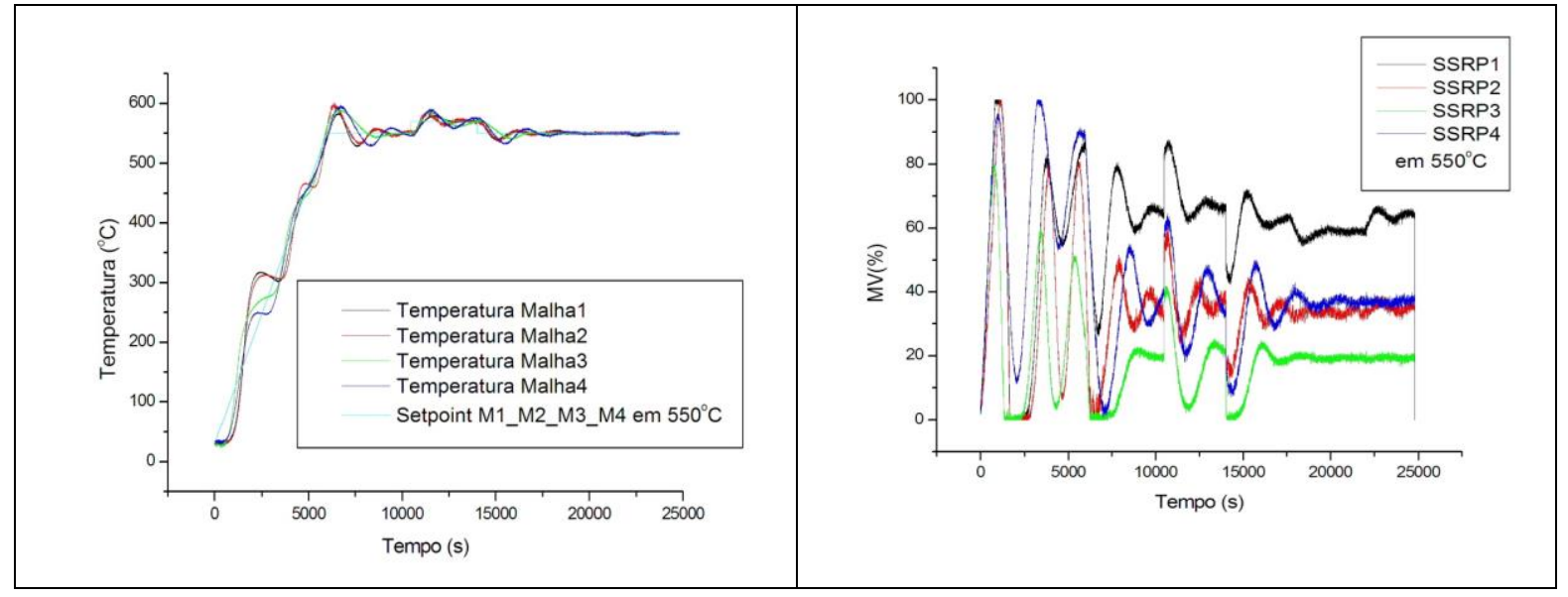

Figura 4: Temperaturas para as malhas de controle e ações de controle para PID fast. 
Os resultados mostram que as regras definidas na lógica fuzzy foram eficientes, proporcionando um ajuste on-line dos parâmetros eficaz. $\mathrm{O}$ consumo de energia nos três casos avaliados permaneceu constante

Tabela 4: Valores dos critérios de erro e consumo de energia $\left(550^{\circ} \mathrm{C}\right)$.

\begin{tabular}{|l|c|c|c|}
\hline \hline Critério de Erro - Malha 1 & PID slow & PIDfuzzy & PID fast \\
\hline \hline$I T A E-$ Servo & $41.569 .015,40$ & $27.027 .797,40$ & $28.883 .982,90$ \\
\hline$I T A E-$ Regulador & $20.417 .879,90$ & $17.497 .134,50$ & $8.804 .600,90$ \\
\hline \hline$I A E-$ Servo & $32.114,50$ & $34.799,80$ & $32.978,40$ \\
\hline$I A E-$ Regulador & $5.683,80$ & $12.345,20$ & $6.197,30$ \\
\hline \hline$I S E-$ Servo & $376.593,73$ & $424.148,52$ & $368.635,56$ \\
\hline$I S E-$ Regulador & $44.795,68$ & $40.883,28$ & $15.064,39$ \\
\hline Consumo de energia $\mathrm{kJ} / \mathrm{kg}$ & $17.058,00$ & $17.121,07$ & $16.743,60$ \\
\hline \hline
\end{tabular}

\section{CONCLUSÕES}

A implementação da estratégia de controle PID com ajuste on-line dos parâmetros mostrou-se viável e de implementação relativamente simples, possibilitando inclusive embarcar a estratégia de controle em hardware. A proposta das regras fuzzy tem gerado um controlador robusto, adaptando-se as adversidades implementadas.

\section{REFERÊNCIAS BIBLIOGRÁFICAS}

ALMEIDA, O. M., COELHO, A. A. R. Controlador PID com Escalonamento Nebuloso dos Ganhos: Auto-sintonia, Análise e Implementação. V Simpósio Brasileiro de Automação Inteligente, V SBAI, 2001

FREITAS, L. T. F., LIMA, O. M., ALMEIDA, O. M., BRAGA, P. S. A. Aprendizado por Reforço no Ajuste PID-Fuzzy Aplicada. 2008

OGATA, K. Engenharia de Controle Moderno. Rio de Janeiro: Prentice Hall, ed.4, 2003

SANDRI, S., CORREA, C. Lógica Nebulosa. V Escola de Redes Neurais, São José dos Campos, São Paulo, 1999

SEBORG, D.E., EDGAR T.F., MELLICHAMP, D.A. Process Dynamics and Control. John Willey, 1989

STEPHANOPOUlos, G. Chemical Process Control, an Introduction to Theory and Practice. Prentice-Hall Inc: New Jersey, 1984

WIGGERS, V. R. Simulação, Projeto e Construção de uma Unidade Piloto de Multi-propósito para Pirólise de Resíduos. (Dissertação de Mestrado), Universidade Estadual de Campinas, Campinas, São Paulo, 2003

ZADEH, L. A. Fuzzy Sets. Information and Control, v.8, p.338, 1965 